\title{
Association between Redundant Nerve Root and Clinical Outcome after Fusion for Lumbar Spinal Stenosis
}

\author{
Lim Joon Yoon ${ }^{1}$, Byung Gwan Moon ${ }^{2}$ In-Suk Bae ${ }^{1}$, Hee In Kang ${ }^{1}$, Jae Hoon Kim¹, \\ Deok Ryeong Kim²
}

'Department of Neurosurgery, Nowon Eulji Medical Center, Eulji University, Seoul, Korea

${ }^{2}$ Department of Neurosurgery, Uijeongbu Eulji Medical Center, Eulji University, Uijeongbu, Korea

Objective: This study aimed to determine the association between redundant nerve root and clinical outcome after fusion for lumbar spinal stenosis by comparing outcomes in patients with or without redundant nerve root.

Methods: A total of 163 patients who underwent spinal fusion surgery between January 1, 2014, and December 31, 2018, were enrolled. Patients were divided into two groups: with a redundant nerve root ( $\mathrm{R}$ group) and without a redundant nerve root ( $\mathrm{N}$ group). The clinical outcome of the two groups was compared using VAS and claudication distance.

Results: A total of 46 patients had a redundant nerve root, while the remaining 117 did not. Symptom duration was significantly different between the two groups $(R=41.0 \pm 25.0, N=24.1 \pm 12.2, p<0.001)$. Changes in the VAS score for lower extremity pain between the two groups at 1 year after surgery showed that patients without a redundant nerve root had significantly better outcomes than those with a redundant nerve root (R: $4.2 \pm 1.3 \mathrm{vs}$. N: $5.5 \pm 1.4 ; p<0.001$ ).

Conclusion: Redundant nerve root on MRI is associated with clinical outcome after fusion for lumbar spinal stenosis. Patients with a redundant nerve root had poor outcomes after fusion surgery for lumbar spinal stenosis than those without a redundant nerve root.

Key Words: Cauda equina; Spinal stenosis; Spinal fusion

\section{INTRODUCTION}

Degenerative lumbar spinal stenosis (LSS) is characterized by

Received: August 27, 2021 Revised: September 11, 2021

Corresponding Author: Byung Gwan Moon

Department of Neurosurgery, Uijeongbu Eulji Medical Center, Eulji

University, 712 Dongil-ro, Uijeongbu 11759, Korea.

Tel: +82-31-951-2760, Fax: +82-31-951-3300,

E-mail: mbg2304@eulji.ac.kr

ORCID: https://orcid.org/0000-0003-4594-1907 narrowing of the spinal canal and constriction of the neural structures, and occurs due to changes in the ligamentum flavum, marked hypertrophy of the facet joints, or both. Depending on the location of the narrowing, patients usually present with intermittent neurogenic claudication symptoms (e.g., buttocks or leg pain while walking or standing that is relieved upon sitting or lumbar flexion) or a more well-defined radicular pain [1]. LSS is the most common reason for lumbar spine surgery in adults older than 65 years [2].

LSS patients with neurological symptoms often present with

(C) 2021 Korean Neuro-Pain Society

(c) This is an Open Access article distributed under the terms of the Creative Commons Attribution Non-Commercial License (http://creativecommons.org/licenses/by-nc/4.0/) which permits unrestricted non-commercial use, distribution, and reproduction in any medium, provided the original work is properly cited. 
a thickened and loop-shaped redundant nerve roots (RNR) of the cauda equina on magnetic resonance imaging (MRI) scans. Studies have shown that the prevalence rates of RNR among patients with LSS can range from $33.8 \%$ to $43.3 \%$ [3-6].

RNR was first reported by Verbiest in 1954 [7] and the term was coined by Cressman and Pawl in 1968 [8]. RNR is a sinusoidal deflection of the cauda equina nerve root, and it is observed on the sagittal plane of T2-weighted MR images. It can also be observed as an enlarged dot sign on the axial plane of T2-weighted MR images. While several studies have shown that the symptoms are worse in patients with RNR, the relationship between RNR and outcome after spinal fusion surgery has not yet been established.

In this study, we aimed to evaluate the clinical significance of RNR on MRI in LSS by comparing patient clinical outcomes after spinal fusion.

\section{MATERIALS AND METHODS}

\section{Study Design}

We retrospectively reviewed patients who underwent spinal fusion for LSS at our hospital between January 1, 2014, and December 31, 2018. Patients were considered eligible for inclusion if they (1) were diagnosed with degenerative LSS, (2) had one-level spinal fusion, (3) had available preoperative MRI of the lumbar spine in the neutral position; and (4) were clinically followed-up for at least 12 months. Patients were excluded if they (1) presented with a lumbar infection before surgery, (2) presented with vertebral compression fracture, (3) underwent fusion of more than 2 levels, or (4) exhibited complex conditions requiring subsequent surgery within 12 months.

All medical records of the included patients (hospital charts and radiologic findings) were retrieved from the institutional databases. Demographic data, medical history, and clinical outcomes were reviewed.

This study was approved by the Institutional Review Board of Eulji University, Korea, and conformed to the tenets of the Declaration of Helsinki. The need for informed consent was waived because of the retrospective nature of the study. All individual records were anonymized before analysis.

\section{Redundant Nerve Root (RNR) Definition and Radio- logic Evaluation}

Two spinal surgeons, blinded to the patient's clinical care, independently assessed each MR image. RNR was identified in the lumbar spine based on apparent tortuosity of elongated and coiled nerve roots in the subarachnoid space on sagittal
T2-weighted MRI. Patients were accordingly divided into two groups: RNR group (R group) and non-RNR group ( $\mathrm{N}$ group) (Fig. 1).

\section{Surgical Technique}

All surgeries were performed by a single surgeon at our institute. The spinal fusion was performed following the surgical technique described by Kim et al. [9], also of our institution. A conventional posterior midline approach was used. Autologous graft materials were harvested from the iliac crest, lamina, and index-level facet joints. The distraction of the disc space was established by hip flexion on the operating table and an Inge laminar distractor. The surgeon limited the use of variable heights of the rimier and disc space elevator to prevent excess endplate damage, as well as disc space distraction to prevent graft subsidence and loss of lumbar lordosis. The surgeon preferred the use of various types of curettes and curved chisels to prepare the disc space. The interbody cage geometry was determined according to the manufacturer's design. Each cage was placed in an axial and parallel direction. Interbody bone grafting is important for compact disc space packing rather than loose filling. The hip flexion surgical posture was returned to a flat prone position to achieve the original lumbar lordosis at the end of the operation. The posterior compression force of the pedicle screw locking system was applied to provide a posterior tension bending force and restore the lumbar lordosis.

\section{Outcome Assessment}

Clinical outcomes were measured using a visual analog scale (VAS) and the distance that causes neurogenic claudication. VAS score was measured before surgery, and 1 month and 1 year after surgery. When assessing the VAS score, patients were asked to rate their pain on a scale from 0 to 10 , with 0 representing no pain and 10 representing the extreme pain. The

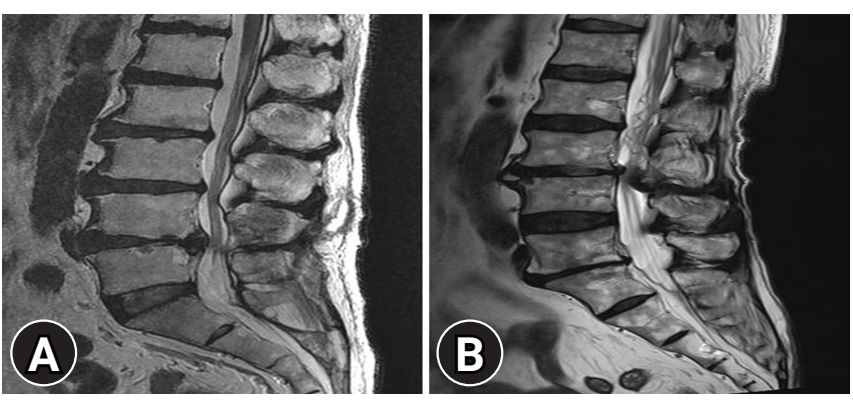

Fig. 1. (A) Sagittal T2-weighted MR image shows a normal distribution of the nerve roots. (B) Sagittal T2-weighted MR image shows RNR in the cauda equine. 
change in VAS score was calculated by subtracting the preoperative score from the postoperative score.

The distance that causes neurogenic claudication in patients was investigated. Patients were classified into four groups according to the distance as follows: very severe, $<100 \mathrm{~m}$; severe, $100-500 \mathrm{~m}$; moderate, $500-1,000 \mathrm{~m}$; and mild, $>1,000 \mathrm{~m}$.

\section{Statistical Analysis}

Continuous variables are expressed as mean $\pm \mathrm{SD}$ or median with interquartile range, while discrete variables are expressed as counts and percentages. The chi-square test and Student's $\mathrm{t}$-test were used to assess the clinical differences between the two groups. VAS and ODI scores were compared using repeated-measures analysis of variance.

Box plots with dot plots were used to visualize the association between the change in VAS scores according to the presence or absence of RNR on MRI. Statistical significance was set at $\mathrm{p}<0.05$. All statistical analyses were performed using $\mathrm{R}$ version 3.5.2 (https:/www.r-project.org/).

\section{RESULTS}

\section{Demographic Characteristics of Patients}

Complete data were available for 163 patients. A total of 46 patients in the R group and 117 patients in the $\mathrm{N}$ group, with a mean age of 64.9 years and 66.0 years, respectively. There were 15 men (32.6\%) in the R group and 41 (35.0\%) in the N group.

While the symptoms surfaced 24.1 months before surgery in group $\mathrm{N}$, the onset period was longer at 41.0 months in group $\mathrm{R}$. The duration of symptoms showed significant differences between the two groups. Table 1 presents a summary of the two groups' characteristics.

\section{Clinical Outcomes}

The VAS score was examined for lower extremity pain, showing significant improvement in both groups. The mean VAS scores in the $\mathrm{N}$ and $\mathrm{R}$ groups were $8.4 \pm 1.1$ and $8.0 \pm 1.3$ before surgery, $3.5 \pm 0.9$ and $3.7 \pm 1.0$ at 1 month after surgery, and $2.8 \pm 1.1$ and $3.9 \pm 1.4$ at 1 year after surgery, respectively.

At 1 month postoperatively, the VAS score changes were greater in the $\mathrm{N}$ group than in the R group; however, this difference was not statistically significant (Fig. 2A). At 1 year after surgery, the $\mathrm{N}$ group showed significantly better pain outcomes than the R group ( $\mathrm{p}<0.001)$ (Fig. 2B).

There was no significant difference in the preoperative breakdown of symptom severity in each group according to the neurogenic claudication distance (Fig. 3A). One year after surgery, patients with mild and moderate claudication group comprised $9 \%$ and $75 \%$ of the $\mathrm{N}$ group, respectively, and $2 \%$ and $22 \%$ of the R group (Fig. 3B).

\section{DISCUSSION}

The purpose of this study was to determine how the presence of RNR on MRI, which is relatively common in LSS patients, is related to postoperative outcomes. In our study, R group showed a longer symptom duration before surgery. In addition, the R group showed poorer outcomes at 1 year after spinal fusion than the $\mathrm{N}$ group.

The clinical significance of RNR in patients with LSS has been scarcely explored. A previous clinical study by Suzuki et al. [10] demonstrated that patients with RNR had advanced age, a longer duration of symptoms, and more severe neurological signs and symptoms. However, there was no significant age difference between the $\mathrm{R}$ and $\mathrm{N}$ groups in our study.

The pathogenesis of RNR has been attributed to a squeezing force from the constricted spinal canal acting on the nerve roots [5]. Mechanical entrapment of nerve roots occurs at the canal stenosis site, restricting their normal mobility [11]. This entrapment causes elongation and coiling of the nerve roots

Table 1. Clinical characteristics of patients receiving spinal fusion for lumbar spinal stenosis

\begin{tabular}{lccr}
\hline Characteristic & Non-RNR group $(\mathrm{N}=117)$ & RNR group $(\mathrm{N}=46)$ & $\mathrm{p}$-value \\
\hline Age & $66.0 \pm 9.0$ & $64.9 \pm 8.7$ & 0.486 \\
Sex (male, \%) & $41(35.0 \%)$ & $15(32.6 \%)$ & 0.911 \\
Height $(\mathrm{cm})$ & $163.1 \pm 5.5$ & $163.6 \pm 6.8$ & 0.591 \\
Weight $(\mathrm{kg})$ & $61.5 \pm 7.1$ & $60.0 \pm 8.0$ & 0.215 \\
BMl & $23.2 \pm 2.7$ & $22.5 \pm 3.3$ & 0.159 \\
Symptom duration (mo) & $24.1 \pm 12.2$ & $41.0 \pm 25.0$ & $<0.001$ \\
Hypertension & $27(23.1 \%)$ & $11(23.9 \%)$ & 1.000 \\
Diabetes mellitus & $9(7.7 \%)$ & $5(10.9 \%)$ & 0.733 \\
\hline
\end{tabular}

RNR: redundant nerve roots, BMI: Body Mass Index. 

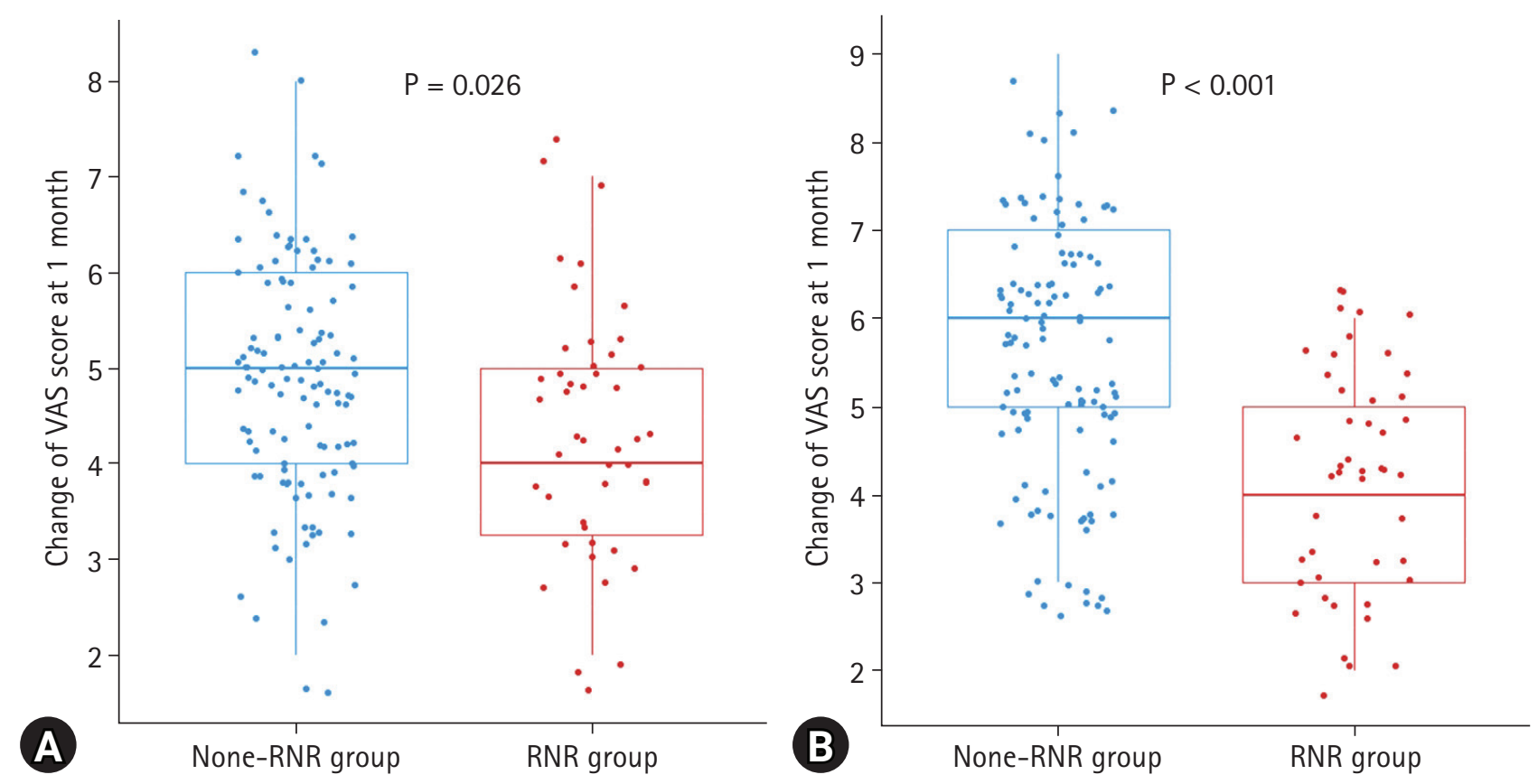

Fig. 2. Comparison of VAS score change for lower extremity pain at (A) 1 month and (B) 1 year after spinal fusion between RNR and non-RNR groups.
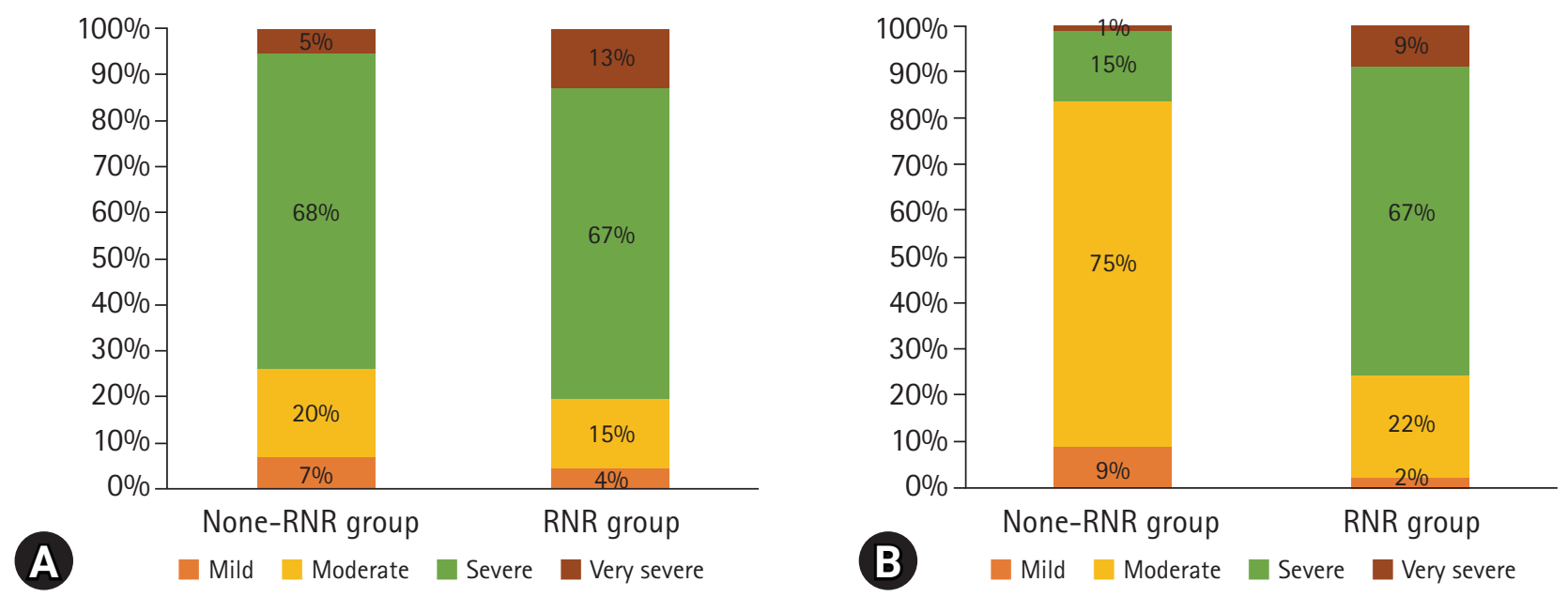

Fig. 3. Bar graph according to the neurogenic claudication distance between the two groups (A) before surgery and (B) 1 year after spinal fusion.

proximal to the stenotic level. Although other terms such as "elongated nerve root" might be more accurate, the term "RNR" has been commonly used. The rate of RNR detection is higher in the extension position than in the flexion or neutral positions [12].

According to Cong et al. [13], RNR is associated with symptomatic LSS, which may be viewed as a potentially powerful prognostic indicator of worse postoperative functional recovery in symptomatic LSS. In addition, in a meta-analysis of seven studies involving a total of 1,046 patients, RNR was find to be a negative prognostic factor after decompression surgery for LSS [14]. Thus, the results of our study are consistent with those of previous studies.

In our study, there was no significant difference in the changes in VAS score between the two groups 1 month after surgery. However, at 1 year after spinal fusion surgery, the $\mathrm{N}$ group showed significantly better results than the R group. The poorer outcomes in patients with RNR after spinal fusion surgery 
might be due to irreversible nerve damage caused by prolonged compression [15-17]. The progression of irreversible neural damage in the R group may have allowed for good progress immediately after surgery, but the symptoms ultimately worsened again with time. In a review of lumbar canal stenosis, Kobayashi [18] reported that such irreversible damage is specific to nerve roots because they are devoid of tic vessels but are immersed in the cerebrospinal fluid (CSF) of the subarachnoid space, where the blood supply remains dependent on peripheral flow (ascending) and flow in the spinal cord's direction (descending).

Our study has several limitations. First, it was a retrospective study and included a relatively small number of patients. Since our study was retrospective, it was more likely to be affected by various types of bias as compared to a randomized controlled study. Second, the decision on spinal fusion was made solely by the surgeon, which might have introduced a selection bias. Third, the outcomes were investigated over the relatively short period of one year. Finally, the results did not definitively show whether the RNR is resolved after surgery since follow-up MRI was not routinely performed. Therefore, a larger, randomized controlled case study and long-term follow-up are required in the future.

\section{CONCLUSION}

RNR on MRI is associated with clinical outcome after fusion for lumbar spinal stenosis. Patients with a redundant nerve root had poor outcomes after fusion surgery for lumbar spinal stenosis than those without a redundant nerve root. Our insights may help physicians decide the surgical plan and predict the outcome after fusion for lumbar spinal stenosis.

\section{CONFLICTS OF INTEREST}

No potential conflict of interest relevant to this article was reported.

\section{REFERENCES}

1. Weinstein JN, Tosteson TD, Lurie JD, Tosteson AN, Blood E, Hanscom B, et al., SPORT Investigators. Surgical versus nonsurgical therapy for lumbar spinal stenosis. N Engl J Med 2008;358:794-810

2. Deyo RA. Treatment of lumbar spinal stenosis: a balancing act. Spine J 2010;10:625-627

3. Chen J, Wang J, Wang B, Xu H, Lin S, Zhang H. Post-surgi- cal functional recovery, lumbar lordosis, and range of motion associated with MR-detectable redundant nerve roots in lumbar spinal stenosis. Clin Neurol Neurosurg 2016;140: 79-84

4. Hur JW, Hur JK, Kwon TH, Park YK, Chung HS, Kim JH. Radiological significance of ligamentum flavum hypertrophy in the occurrence of redundant nerve roots of central lumbar spinal stenosis. J Korean Neurosurg Soc 2012;52:215

5. Suzuki K, Takatsu T, Inoue H, Teramoto T, Ishida Y, Ohmori K. Redundant nerve roots of the cauda equina caused by lumbar spinal canal stenosis. Spine (Phila Pa 1976) 1992;17:1337-1342

6. Zheng F, Farmer JC, Sandhu HS, O'Leary PF. A novel method for the quantitative evaluation of lumbar spinal stenosis. HSS J 2006;2:136-140

7. Verbiest $H$. A radicular syndrome from developmental narrowing of the lumbar vertebral canal. J Bone Joint Surg Br 1954;36:230-237

8.Cressman MR, Pawl RP. Serpentine myelographic defect caused by a redundant nerve root. Case report. J Neurosurg 1968;28:391-393

9. Kim JK, Moon BG, Kim DR, Kim JS. Postoperative flat back: contribution of posterior accessed lumbar interbody fusion and spinopelvic parameters. J Korean Neurosurg Soc 2014;56:315-322

10. Suzuki K, Ishida Y, Ohmori K, Sakai H, Hashizume Y. Redundant nerve roots of the cauda equina: clinical aspects and consideration of pathogenesis. Neurosurgery 1989;24: $521-528$

11. Rigsby CM, Virapongse C, Duncan C. Positional variability in redundant lumbar nerve-root syndrome. Surg Neurol 1983;19:513-516

12. Murphy RW. Nerve roots and spinal nerves in degenerative disk disease. Clin Orthop Relat Res 1977;(129):46-60

13. Cong L, Zhu Y, Yan Q, Tu G. A meta-analysis on the clinical significance of redundant nerve roots in symptomatic lumbar spinal stenosis. World Neurosurg 2017;105:95-101

14. Marques CJ, Hillebrand H, Papavero L. The clinical significance of redundant nerve roots of the cauda equina in lumbar spinal stenosis patients: a systematic literature review and meta-analysis. Clin Neurol Neurosurg 2018;174:40-47

15. Kondo A, Koyama T, Ishikawa J, Hanakita J, Iwaki K. Redundant nerve roots of the cauda equina. Acta Neurochir (Wien) 1978;42:209-215

16. Ono A, Suetsuna F, Irie T, Yokoyama T, Numasawa T, Wada $\mathrm{K}$, et al.. Clinical significance of the redundant nerve roots of the cauda equina documented on magnetic resonance 
imaging. J Neurosurg Spine 2007;7:27-32

17. Tsuji H, Tamaki T, Itoh T, Yamada H, Motoe T, Tatezaki S, et al.. Redundant nerve roots in patients with degenerative lumbar spinal stenosis. Spine (Phila Pa 1976) 1985;10:72-82
18. Kobayashi S. Pathophysiology, diagnosis and treatment of intermittent claudication in patients with lumbar canal stenosis. World J Orthop 2014;5:134-145 